Чистякова В.О.

\title{
Анализ экрана в аспекте медиальности (перспективы screen studies)
}

\begin{abstract}
Аннотация: Предметом исследования выступает экран как формообразующий принцип в культуре, а также отдельные воплощения экрана. При всем многообразии современных экранов, гигантских и малых, приватных и публичных, интерактивных и линейных, динамичных и статичных, нами легко узнается экранная форма как таковая: она довольно четко отграничена от визуальных явлений другого порядка. Экран узнается по нескольким признакам: по наличию у него рамки (видимой границы между ним и прочими частями пространства), по наличие поверхности, по демонстрируемой им диалектике сокрытия и обнаружения и по свойству полиэкранности. Последнее из свойств выводит нас на иной уровень анализа экрана: в аспекте медиального. Экран предстает как частный случай медиального, в отношении которого можно только частично реконструировать его «движение», данное нам в момент его «изменения. $B$ исследовании используются подходы media studies, a также методы screen studies, или screenology, рассматривающих экран как «средство» (medium), то есть множественность условий, в том числе материальных, связанных с характеристиками конкретного “носителя» (но не сводимых к ним), которые позволяют состояться видению. Новизна исследования заключается в том, что впервые понятие медиальности было применено к феномену экрана, что позволило объяснить эффект видения, объектом которого выступает изображение как существующее в гранищах того или иного конкретного экрана. В качестве дискуссионного был поставлен вопрос о материальном характере медиума (тетахи), равно как и о степени связанности с материальным медиальности как таковой. Был сделан вклад в разработку перформативного понимания медиального (иными словами, понимания медиа как практики). Был сделан вывод о том, что экран есть частный случай медиального, у которого нет онтологии и которое уклоняется от хронологической определяемости. В этом смысле экран не существует, а «находится в становлении».
\end{abstract}

Review: The subject under research is the screen as a form-making principle in culture as well as particular forms of screen art. Even though there is a great variety of modern screens, gigantic and small, private and public, interactive and linear, dynamic and static ones, we can still easily recognize the screen form as it is. It is clearly distinctive from other visual phenomena. The screen can be recognized based on several features: it has the frame (visible border between the screen and other space), surface, it demonstrates the dialectics of the hidden and discovered and has the polyscreen feature. The latter takes us to a completely new of the analysis of screen, i.e. in terms of mediality. The screen is a particular case of mediality in which regard we can only partly reconstruct the 'movement' given at the moment of 'change'. In her research the author uses the approaches of media studies as well as screen studies or screenology viewing screen as a medium, i.e. the combination of many conditions related to the features of a particular data storage device (but not limited to them) allowing to make information visible. The novelty of the research is in the fact that the author is the first one to apply to the concept of mediality to the phenomenon of screen. The author's approach allows to explain the vision effect when the image is rendered through a particular screen. The author also raises a debating question about the material nature of medium (metaxu) and the relation between the material and mediality. The author has made a great contribution to the development of the performance concept of mediality (in other words, the concept of mediality as a practice). It is concluded that the screen is a particular case of mediality. It has no ontology or chronological certainty. In other words, the screen does not exist but 'being established'.

Ключевъе слова: Экран, медиальное, медиум, экранная культура, экранная практика, телевидение, компьютер, кинематограф, поверхность, граница.

Keywords: Screen, medial, medium, screen culture, screen practice, TV, computer, cinematograph, surface, border. 
$\mathrm{H}$ аправление исследований, получившее в последнее время наименование screen studies, а также, в терминологии Эркки Хухтамо, screenology ${ }^{1}$, довольно четко локализует свой предмет, выделяя из безбрежного океана «медиа» вполне конкретную узнаваемую визуальную форму: экран. При всем многообразии современных экранов, гигантских и малых, приватных и публичных, интерактивных и линейных, динамичных и статичных, нами легко узнается экранная форма как таковая: она довольно четко отграничена от визуальных явлений другого порядка. Экран узнается по нескольким признакам: в первую очередь, по наличию у него рамки (видимой границы между ним и прочими частями пространства). Как пишет Лев Манович, рамка разделяет два абсолютно разных пространства, которые каким-то образом сосуществуют. Этот феномен определяет экран «в самом общем смысле», представляя его в виде некоего виртуального пространства, находящегося «внутри» обыденного пространства ${ }^{2}$. Другая значимая характеристика экрана - это наличие поверхности. Хухтамо, предлагая создать новую область исследований, которую можно было бы назвать «экранологией», определяет экран как «информационную поверхность» ${ }^{3}$. Вилем Флюссер, рассуждая о фотографии как двумерном изображении (плоскости), пишет следующее: «Образы - это означающие поверхности. Они указывают - чаще всего - на нечто «во внешнем» пространстве-времени, что они обязуются представить нам как абстракцию (как сокращение четырех размерностей пространства-

\footnotetext{
${ }^{1}$ Huhtamo E. Elements of Screenology: Toward an Archaeology of the Screen // ICONICS: International Studies of the Modern Image. 2004. Vol. 7. P. 31-82.

${ }^{2}$ Подробнее об этом см.: Манович Л. Археология компьютерного экрана // Экранная культура. Теоретические проблемы: сб. статей / СПб.: «ДМИТРИЙ БУЛАНИН», 2012. Целью настоящей статьи не является рассмотрение конкретных геометрических форм, которые может принимать экран - прямоугольных, квадратных, овальных, «портретных» или «пейзажных», и т.п. На эту тему много писали Л. Манович и Э.Хухтамо, предложившие свои «генеалогии» экранных форм, а также можно привести статью Чарли Гира о происхождении компьютерного экрана: Gere Ch. Genealogy of the computer screen // Visual Communication. 2006. No. 5. DOI: 10.1177/1470357206065306. Режим доступа: http://vcj.sagepub.com/cgi/content/ abstract/5/2/141

${ }^{3}$ Huhtamo E. Op. cit.
}

времени до двумерной поверхности). Эту специфическую способность к абстрагированию поверхностей из пространства-времени и к проецированию обратно в пространствовремя можно назвать “воображением"»4. И зачастую именно воображение становится ключевым моментом при исследовании отдельных разновидностей экрана ${ }^{5}$.

Третьим, наименее изученным, признаком экранной формы является демонстрируемая им диалектика сокрытия и обнаружения. Сам термин «экран» (screen), если обратиться к Oxford English Dictionary, появляется в $\mathrm{XIV}-\mathrm{XV}$ вв. и используется для обозначения «приспособления, служащего защитой от каминного тепла или сквозняков» ${ }^{6}$. При этом этимология слова до сих пор не прояснена окончательно. Как пишет Э.Хухтамо, «экран обычно означал напольный предмет мебели, состоящий из куска легкого, зачастую полупрозрачного материала (бумаги, какого-нибудь вида ткани и т.д.), натянутого на деревянную раму (или на несколько соединенных раздвижных рам)»7. Помимо выполнения своих обычных функций, экраны, как свидетельствует Century Dictionary and Cyclopedia (1911), предназначались для ограждения пользователя от «прочих неудобств или опасностей, защиты от наблюдения, для

${ }_{4}$ Флюссер В. За философию фотографии / Пер. с нем. Г. Хайдаровой. - СПб.: Изд-во С. - Петерб. ун-та, 2008. Стр. 6.

${ }^{5}$ Например, согласно классификации Льва Мановича, существует такая разновидность, как «динамичный» экран, который представлен кинематографом, телевидением и видео. Он может передавать изображение, меняющееся во времени, то есть изображение в движении. К специфическим свойствам, в частности, кинематографа, можно отнести определенный просмотровый режим, требующий от зрителя максимальной вовлеченности в происходящее на экране, при этом рамки экрана как будто не существует вовсе, а точнее - она не замечается (киноэкран просто заканчивается у своих границ, тогда как, например, в случае телевидения рамка гораздо ощутимей). Зритель идентифицирует себя с происходящим на экране: в определенном смысле, кино смотрят воображением. См.: Манович Л. Археология компьютерного экрана // Экранная культура. Теоретические проблемы: сб. статей / СПб.: «ДМИТРИЙ БУЛАНИН», 2012.

${ }^{6}$ Oxford English Dictionary, II edition / ed.by J.A. Simpson and E.S.C. Weiner. Oxford: Clarendon Press, 1989.

7 Хухтамо Е. Элементы экранологии: к проблеме археологии медиа / Экранная культура. Теоретические проблемы: сб. статей / СПб.: «ДМИТРИЙ БУЛАНИН», 2012. Стр. 123. 
маскировки, предотвращения лицезрения посторонними, или для обеспечения личного пространства» ${ }^{8}$. Тем самым, экран мог представлять собой предохраняющую поверхность, препятствующую чему-либо некомфортному или же вторжению в личное пространство. Хухтамо упоминает также интересную разновидность карманного размера экранов для дам. Кроме своего основного назначения, подобные экраны служили предметами моды. «Лицо, прикрытое ручным экраном, пробуждало желание и любопытство, подобно маске, а сокрытие и раскрытие были непреложными компонентами этого “сценария” »9. Кроме того, Хухтамо, проделывая в полном смысле археологическую работу в поисках ранних форм медиа (собственный проект он так и называет: «археология медиа»), делает акцент на крайне важной для понимания природы и социальной функции экрана практике: представлениях Фантасмагории ${ }^{10}$. В этих шоу экран перестает быть часть домашней жизни и переходит в мир публичных увеселений. Его используют, стараясь сделать невидимым (чаще всего путем увлажнения), чтобы «растворить» границы между пространством публики и миром фантазий и чудес; и он же служит завесой, скрывающей секретные трюки и машинные приемы, что способствует иллюзии появления необыкновенных существ «как по волшебству». Так, Фантасмагория впервые наглядно продемонстрировала двойственность процессов сокрытия и обнаружения, присущих затем экрану на всех этапах его существования. В эпоху аудиовизуального (звукозрительного) экрана актуализируется феномен «закадрового пространства» - того, что «экранизации» не подлежит, что всегда остается «за кадром». Можно даже сказать, что закадровое пространство «выраженно отсутствует» в пространстве кадра: кадр есть знак этого постоянного сиюминутного (име-

\footnotetext{
8 The Century Dictionary and Cyclopedia (CDC). New York: The Century Co, revised and enlarged, 1911 (orig. 1889). Vol. VIII, "screen".

${ }^{9}$ Хухтамо Э. Op. cit.

${ }^{10}$ Фантасмагория - публичное представление, популярное с конца XVIII в., во время которого зрителям показывали картинки, в большинстве своем изображавшие чудовищ, призраков и привидения. Они демонстрировались на полупрозрачном экране. Создавался эффект увеличения и уменьшения фигур. См.: Levie, Françoise. Étienne-Gaspard Robertson, la vie d'un fantasmagore, Longueuil et Bruxelles, Les Éditions du Préambule et Sofidoc, coll. «Contrechamp», 1990.
}

ются в виду кинематографические «здесь и сейчас») отсутствия. В определенном смысле, экран есть визуализация отсутствия. И здесь вновь следует вспомнить о роли воображения в деле восприятия экранных произведений/сообщений: именно оно конструирует образ отсутствующего. Ингмар Бергман замечал, что восприятие кинокартины если на что-то и похоже, то только на восприятие музыки: «экспрессивный ряд», минуя волю и интеллект, напрямую воздействует на воображение ${ }^{11}$. Иными словами, кинореальность увлекает нас на такой путь, который во многом создается нашим же воображением, нами же. В этом смысле экран прозрачен: он есть то, что мы «за» ним увидим. Можно предположить, что экран в определенном смысле «трансцендентален»: посредством него (и только посредством него) можно увидеть то, что «за» ним, сам же он как будто «невидим». Он невидим в точно таком же смысле, в каком невидимым является само по себе воображение. Увидеть можно только его предмет, но никак не само воображение. (Так, непрозрачная поверхность киноэкрана создает достаточную прозрачность экранной реальности, чтобы можно было говорить о кино как особом способе существования поэтического)

Наконец, последний из важнейших признаков экрана (в чем также содержится его «экранность») состоит в том, что экран стремиться представлять собой одновременно множество экранов: сама экранная форма как будто неустойчива и тяготеет к полиэкранности. Это свойство находит свое буквальное выражение, к примеру, в экспериментах отдельных телекомпаний, преследующих цель занять лидерские позиции на мировом рынке информации и информаторов. «Об изобилии телевизионной информации, обрушивающейся на современного человека, твердят постоянно и повсеместно. Аудиовизуальная техника очень быстро развивается, и за несколько последних лет благодаря успеху цифровых технологий и переводу на этот стандарт национальных телевизионных систем меняется сам имидж телевидения. Если раньше это был экран включенного телевизора с одной картинкой, то теперь это МОЗАИЧНЫЙ экран. При включении цифрового телевизора на экране возникает мозаика картинок из всех принимаемых программ одновременно, а уже

${ }^{11}$ См.: Бергман И. Латерна магика. М.: Искусство, 1989. 
затем сам зритель выбирает из них либо одну, заполняющую весь экран, либо на фоне «основного» зрелища размещает еще один или несколько прямоугольников с другими программами, за которыми либо будет следить боковым зрением либо время от времени отвлекаться на них от основной программы. Именно такой мозаичный экран стал товарным знаком самой мощной мировой телекомпании $\mathrm{CNN}$, претендующей на роль главного мирового информатора» ${ }^{12}$.

Лев Манович выделает отдельные стадии в истории развития экрана, при этом в хронологическом плане самой последней формой выступает экран компьютера. Именно он тяготеет к тому, чтобы демонстрировать не отдельное изображение, а ряд «окон». «Фактически с момента появления в 1984 году первого компьютера Макинтош сосуществование ряда взаимонакладывающихся окон стало основополагающим принципом современного компьютерного интерфейса. Ни одно из окон не владеет целиком вниманием зрителя. В этом смысле возможность одновременно видеть несколько изображений, которые сосуществуют на одном экране, можно сравнить в феноменом заппинга - быстрого переключения телевизионных каналов, позволяющего зрителю следить за несколькими програмами» ${ }^{13}$.

Такого же рода эксперименты можно найти в практике игрового кинематографа. Прием мозаичного изображения используется время от времени в том или ином кино- или телефильме. Помимо этого, в истории экранной культуры имели место попытки одновременной трансляции двух и более сюжетных линий одного произведения, соответственно, с двух и более экранов. «В послевоенные годы поиски в этом направлении связывались часто с использованием так называемого полиэкрана - комбинации нескольких самостоятельных изображений, одновременно проецируемых на отдельные экраны или части большого экрана... Известно, что еще в 1914 году Я. Протазанов в фильме «Драма у телефона» разделил кадр на три части, поместив в каждую из них особое изображение; три одновременно происходивших и связанных между собой общим сюжетом действия

\footnotetext{
${ }^{12}$ Иоскевич Я.Б. Новые технологии и эволюция художественной культуры. Спб.: РИИИ, СПбГУКит, 2003. Стр. 143.

${ }_{13}$ Манович Л. Археология компьютерного экрана // Экранная культура. Теоретические проблемы: сб. статей / СПб.: «ДМИТРИЙ БУЛАНИН», 2012. Стр. 58.
}

демонстрировались одновременно... В 1927 году во Франции как художественная сенсация был воспринят выпуск фильма «Наполеон» А.Ганса, изображения которого одновременно проецировались на три расположенных в форме триптиха экрана. Однако, несмотря на выдающиеся достоинства фильма, публика не приняла полиэкранный способ показа, и Гансу пришлось перемонтировать его для показа на обычном экране» ${ }^{14}$

Таким образом, тяготение экрана к существованию в режиме «полиэкранности» выражается, по меньшей мере, двумя способами. Во-первых, это описанный выше принцип мозаичности, делающий возможным проецирование нескольких изображений либо на несколько отдельных экранов, либо в виде отдельных частей одного и того же экрана. А во-вторых, это постоянно увеличивающаяся численность разновидностей экранных форм, рост которой связан прежде всего с тем, что экранную форму начинают принимать такие сферы человеческой жизнедеятельности, которые ранее к экранной культуре могли не иметь вовсе никакого отношения. В качестве примера можно привести трансформации, произошедшие не так давно с понятием музейной базы данных: «...электронная экспозиция - не просто интерфейс, дающий посетителю доступ к электронному каталогу. Она имеет собственный сценарий. Кроме того, ряд материалов, как и в других электронных изданиях, готовится специально для данной публикации: тексты, изображения, аудио-видеоклипы, специальные мультимедиа-программы для посетителей (в том числе игры) и др. Но по сравнению с CD-ROM, веб-сайтом, локальным информационным киоском электронная экспозиция обладает очевидным преимуществом: она имеет механизм синхронизации. Основную часть электронной экспозиции составляют материалы из музейной БД (базы данных - В.Ч.), и все изменения и дополнения, сделанные хранителем в рабочей базе, немедленно визуализируются в системе» ${ }^{15}$ (Лебедев А.В. - В.Ч.). В связи с тем, что именно экран становится средством доступа к любой информации, на него опираются работники самых разных профессиональных сфер (экраны диспетчеров, операторов, секретарей, инженеров, врачей, сотрудников

\footnotetext{
14 Гинзбург С.С. Очерки теории кино. М.: Искусство, 1974. Стр. 25-26.

${ }_{15}$ Основы музееведения. Учебное пособие. М.: Едиториал УРСС, 2005. Стр. 473.
} 
служб безопасности, пилотов и т.п.), а также люди в ходе своей повседневной жизни (экраны банкоматов, дисплеи самых различных приборов и, конечно, компьютерный экран). Механизмы экранного представления данных преобразовали многие области практики, в том числе научной. Это касается, например, этнографии, чей метод основывается на непосредственном наблюдении: возможности фото-, кино- и видеосъемки с момента своего возникновения находились под пристальным вниманием со стороны ученых, усмотревших в ней новые способы непосредственного наблюдения за объектом исследования. В рамках физической географии роль экранного научного и научно-технического документа серьезно возросла, начиная с 60-80-х гг. прошлого века, когда появились космические и геоинформационные методы исследования. Помимо этого, фото-, кино- и видеодокумент в ряде случае является очень важным компонентом географической экспедиционной практики (в частности, фотография как полевой документ функционирует в качестве способа получения информации о ландшафте, при его описании и изучении). Исследование поверхности Земли и природных ресурсов при помощи воздушных, космических и наземных съемок практикуется в географии уже в течение нескольких десятилетий. Более того, в настоящий момент существует целая система средств и способов получения и обработки результатов аэрокосмических и наземных съемок для решения научных и прикладных задач в самых различных областях инженерной и научной деятельности. Эти результаты активно используются в геодезии и картографии, в исследованиях природных ресурсов Земли и космического пространства, в экологии, геологии, в строительстве, архитектуре и археологии, в сельском и лесном хозяйстве, в астрономии, медицине и т.д. В этом можно усмотреть подтверждение тезиса М. Маклюэна о том, что «средство есть сообщение»: используемые средства влияют на результат деятельности, как бы «впечатываясь» в него и становясь его составной частью. Технологии, ведущие к созданию экранной формы, меняют предмет деятельности таким образом, что им становится уже не естественный (в том или ином смысле) объект, а экранный документ. Так, географ имеет дело со сгенерированными на экране данными - скорее, именно они теперь его предмет, а не «природный ландшафт».
Экран тем самым содержит в себе, как в зародыше, незавершенное множество самых различных экранных воплощений, в связи с чем его хочется назвать не столько формой, сколько формообразующим принципом в культуре.

Для экрана существует еще и третий путь создания ситуации «полиэкранности». Не исключено, что этот путь и есть самое «экранное», в том, что представляет собой экран. То есть в том, что представляет собой экран как таковой, вне зависимости от того, что на нем изображается в текущий момент времени (если, конечно, уместно рассуждать об экране вне какого-либо экранного изображения). В повседневной практике довольно часто встречаются экраны, на которых ничего не изображено: погасшие или еще не включенные экраны, «мертвые» экраны, испорченные экраны, пустые экраны, «слепые» экраны, белые экраны, черные экраны. Своего рода «анти-экранную» выставку провел один из классиков видеоарта Нам Джун Пайк в Германии в 1963 году. В павильоне были расставлены десятки телевизоров, часть из них была перевернута, и все они показывали картинку с искажением. Телевизоры исполняли здесь анти-телевизионную, по сути, функцию: транжирили минуты экранного времени, демонстрируя изображение, начисто лишенное признаков знакомой телевизионной картинки (не содержало ли это зрелище одно из крайних выражений эстетики телевизионного типа?).

Возникает вопрос, что есть экран помимо того, что на нем изображается? Он есть совершенно особого рода поверхность, на которой, даже в отсутствие какого бы то ни было изображения, содержится изображение самой возможности изображения. В этом смысле любая поверхность, предназначенная для изображения (экран телевизора, холст или лист бумаги), не может быть совершенно свободной от изображения. Так как она неизбежно изображает текущее не-изображение как место, в котором изображение может возникнуть. Экран (неважно, выключенный или работающий) при ближайшем рассмотрении оказывается обладающим свойством визуализировать возможность: выключенный экран - саму возможность изображения, работающий - целый спектр возможностей, не для каждой из которых существует соответствующая действительность. Неработающий экран репрезентирует возможность, благодаря которой нечто становится возможным (как правило), а экран работающий репрезентирует 
(в том числе) возможность, благодаря которой невозможно ничего. Точнее, невозможно то, чья возможность «налицо», то есть визуализирована. Но если в опыте невозможно то, возможность чего мы «видим», то это означает, что перед нами «пустая» возможность, видимость возможности (или возможность в виде ее «видимости»). «Вижу, что возможно, но - невозможно». Оказывается, что «А» не равно «А», в нарушение закона тождества. Диссоциация, возникающая между «А» и «A», обязана своим появлением этому самому «я вижу», «видению», «вижу «А» (но не «А»), то есть «замеченной» возможности (того, чего нет в действительности), а еще точнее - самому «замечанию» этой возможности как возможности. Как один из примеров - (не)возможность оказаться «по ту сторону» экрана, шагнуть в «заэкранье», как в «зазеркалье». Или шагнуть с экрана в зал. Хотя, пока существует экран, возможность такого «перехода» существует визуально. Впервые с этой «возможностью без действительности» столкнулись зрители первого публичного киносеанса, организованного братьями Люмьер в Париже 28 декабря 1895 года, - день, ставший официальной датой рождения кино. По легенде, зрители «Прибытия поезда», увидев надвигающийся на них с экрана поезд, начали в панике выбегать из зала. Так, впервые была «увидена» возможность перехода экранного изображения в мир «по эту сторону экрана». Возможность увидена (усмотрена), но нет в действительности того, чьей возможностью данная возможность является. А сама возможность, тем не менее, «является» (от глагола «являться»).

С 28 декабря 1898 года отсчитывает свою жизнь «двойник» настоящего времени, то есть, по сути, некий «параллельный мир»: параллельные «здесь и сейчас», происходящие «на» экране как «за» экраном. Звукозрительный ряд кино представляет собой не что иное, как альтернативные «здесь и сейчас», своего рода копию настоящего времени. «... изобретение кинематографа направлялось тем же самым мифом, который подспудно определял все остальные разновидности механического воспроизведения реальности, увидевшие свет в XIX веке, - от фотографии до фонографа. Это - миф интегрального реализма, воссоздающего мир и дающего такой его образ, который неподвластен ни свободной интерпретации артиста, ни необратимому ходу времени. Если кинематограф при своем рождении не обладал всеми атрибутами тотального кино, то лишь потому, что феи, стоящие у его колыбели и стремившиеся оделить его всеми дарами, были недостаточно сильны в техническом отношении» ${ }^{16}$. Чем же в этой ситуации «двумирия» является сам по себе экран? Очевидно, что он выступает своеобразным «окном» в этот «параллельный мир» (советский киновед Ростислав Юренев именно так и озаглавил свой труд, подготовленный для широкого круга читателей, - «Чудесное окно» ${ }^{17)}$ ). Недаром в отношении экрана часто применяется метафора «окна» (в другой мир, в иное измерение). Вилем Флюссер отмечает также, что экранам присуще некоторые свойства дверей: они как будто позволяют нам «войти» в ту область, которую изображают ${ }^{18}$. Это касается не только интерактивных экранов: такая трактовка позволяет отнести к категории экранной формы широкий диапазон явлений, включая живопись и другие разновидности визуальной художественной культуры.

Так, экранная культура создает ситуацию полиэкранности не только рассмотренными выше способами, - с помощью принципа мозаичности изображения и наделяя экранной формой то, что раньше ее не имело - но и, что самое важное, при помощи присущей экрану «функции окна», то есть окна в некий «параллельный мир»- результат работы «мифа тотального реализма», как его определил Андре Базен. Этот «мир» дан нам через «окна», которые на него «выходят», - так же, как окна гостиной выходят в сад. Эпоха экранной культуры подразумевает множество «окон» именно такого рода - организующих для нас видимость другого «мира» (или «миров»). В современной экранной практике (особенно в области телевизионной рекламы, в теле- и видеозаставках, видеоклипах) функция «окна», принадлежащая экрану как таковому, используется на уровне технического приема, призванного осуществлять зрительный переход из одного пространства в другое. Кроме того, «многомирие», переходы из мира в мир - одна из самых популярных сюжетных линий и тем,

${ }^{16}$ Базен А. Что такое кино? Сб. статей. М.: Искусство, 1972. Стр. 51.

${ }_{17}$ Юренев Р.Н. Чудесное окно: Краткая история мирового кино: Кн. для учащихся. - М.: Просвещение, 1983.

${ }^{18}$ Flusser $V$. Two approaches to the Phenomenon, Television / The New Television: A Public/Private Art / ed.by D.Davis and A. Simmons, Cambridge, Mass.: The Mitt Press, 1977. 
используемых в игровом кино и компьютерных играх последних десятилетий. Не случайно, что в контексте экранной культуры получает интенсивное развитие проблематика, связанная с феноменом виртуальной реальности.

В целом, можно констатировать, что в момент возникновения «альтернативного настоящего времени» (момент, разделивший мир и «мир» и установивший между ними «окно»), на свет появился экран как «полиэкран», поскольку свойство «от-делимости», открытое однажды в отношении мира, будет отныне миру сопутствовать и творить «N-мирие». Экран же будет выступать тем, что отделяет «мир» от «мира» и дает возможность «увидеть» - из одного «мира» другой «мир».

Тем самым «экранная культура» подразумевает множество «дверей-окон», за каждым из которых мы видим то, что видим. И даже если перед нами «мертвый» экран, то мы видим в нем возможность того, что способно обрести статус существующего в нашем видении. В этой ситуации неважно, имеем ли мы дело с экраном как явлением художественной культуры (кино- или телефильм, видеоклип и т.п.), не предполагающим ответного воздействия со стороны зрителя, или экраном как форматом интерактивного общения (дисплей мобильного телефона, монитор компьютера или ноутбука и т.д.). Экран в любом случае выступает своего рода «окном» как способом визуальной организации того содержания, которое рамками данного «окна» ограничено. Это означает, что экран как «средство» (medium) представляет собой множественность условий, в том числе материальных, связанных с характеристиками конкретного «носителя» (но не сводимых к ним), которые позволяют видению состояться. Речь идет о превращении чего-то во что-то, что видно, посредством чего-то еще: Дитер Мерш исследует в этом случае роль предлога посредством/через, который в латинском языке соответствует предлогу Per, а в греческом - предлогу Dia. «Оказывается, что роль эвристически перечисленных приставок и предлогов для медиального действительно значительна: так per-sona указывает на медиальность маски, которая, то закрывая, то открывая, заставляет лицо говорить звуками - «через» (per) плюс «звук» (sono), в то время как пер-спектива дословно означает «взгляд сквозь/через» (Дюрер) $)^{19}$

19 Сравн.: Erwin Panofsky, Die Perspektive als ,symbolische Form', in: ders., Aufsätze zu Grundfragen der Kunstwissenschaft, Berlin 1998, S. 99. Подобное мы и этот взгляд мало зависит от прозрачности среды, в этом «через/сквозь» скорее опознается та математическая структура, которую предлагает вид. Речь идет о применении математической традиции, которая задает нашему зрению определенные направление и угол и «через» это конструирует видимость» ${ }^{20}$. Медиальность экрана позволяет состояться видению, объектом которого выступает изображение как существующее в границах того или иного конкретного экрана. Мерш обращается к эстетике Аристотеля, при переводе которой на латинский исторически возникло понятие «медиа», а также к теориям перформативности, которые вносят в медиальное параметр практического. «... в разделе, где речь идет о восприятии и зрении, он (Аристотель - В.Ч.) пишет о центральном месте Metaxu - «чегото, лежащего между», что помогает установить контакт между глазом и объектом, чтобы быстро перейти к тому понятию, которое зародилось в более старых теориях восприятия: диафаническое, или «просвечивание, проблеск» (diaphaino) практически синонимично пер-спективе. ... диафаническое обозначает то, что, что дает возможность появиться - diaphaino обозначает «появление» (phaino), проявление через что-то, и это что-то имеет для Аристотеля материальный характер» ${ }^{21}$. Дискуссионным остается вопрос о материальном характере медиума (metaxu), равно как и о степени связанности с материальным медиальности как таковой. Дитер Мерш делает здесь шаг к перформативному пониманию медиального (иными словами, к пониманию медиа как практики). В области исследований экрана и его конкретных материальных

находим и у Hans Belting, Florenz und Bagdad. Eine westöstliche Geschichte des Blicks, München 2. Aufl. 2008, S. 23. Слово «перспектива» засвидетельствовано уже у Боэция, но четкое толкование с уклоном в медиальное и образность оно получает только у Дюрера и обозначает «сквозной вид» на ставший видимым мир. Альберти связал это с метафорой окна: окно, его обрамление, высвобождает взгляд, дает нам возможность видеть, открывая свое собственное изобразительное пространство. Можно сказать: перспективы образуют перформативные образные акты; пространство являет собой не просто набор мест и знаков, а нечто, что исследуется и покоряется с помощью движения глаза.

${ }^{20}$ Мерш, Дитер. Мета/Диа. Два различных подхода к медиальному // Исследовательский центр медиафилософии, режим доступа: http://mediaphilosophy.ru/ biblioteca/articles/mersh_dia/\#footnote-27

${ }^{21}$ Мери, Дитер. Там же. 
разновидностей также был сделан шаг в сторону практики: было введено понятие «экранной практики», его ввел Чарльз Массер, чьей целью было помещение конкретной разновидности экранной культуры (он исследовал немое кино) в контекст предшествующих зрелищ, включая проецируемы на экран изображения ${ }^{22}$. Можно сказать, что экран есть частный случай того медиального, у которого, по мысли Мерша, нет онтологии, которое уклоняется от хронологической определяемости и в отношении которого можно только частично реконструировать его «движение», данное нам в момент его «изменения. В этом смысле экран не существует, а «находится в становлении».

\section{Библиография:}

1. GereCh.Genealogyofthecomputerscreen//VisualCommunication.2006.No.5.DOI:10.1177/1470357206065306. Режим доступа: http://vcj.sagepub.com/cgi/content/abstract/5/2/141

2. Huhtamo E. Elements of Screenology: Toward an Archaeology of the Screen // ICONICS: International Studies of the Modern Image. 2004. Vol. 7.

3. Flusser V. Two approaches to the Phenomenon, Television / The New Television: A Public/Private Art / ed.by D.Davis and A. Simmons, Cambridge, Mass.: The Mitt Press, 1977.

4. Levie, Françoise. Étienne-Gaspard Robertson, la vie d'un fantasmagore, Longueuil et Bruxelles, Les Éditions du Préambule et Sofidoc, coll. «Contrechamp», 1990.

5. Musser, Charles. Emergence of the Cinema: the American screen to 1907 // History of the American Cinema. Berkeley; Los Angeles: University of California Press: 1994. Vol. 1. Chapter 1, "Towards a History of Screen Practice".

6. Oxford English Dictionary, II edition / ed.by J.A. Simpson and E.S.C. Weiner. Oxford: Clarendon Press, 1989.

7. Panofsky, Erwin. Die Perspektive als ,symbolische Form', in: ders., Aufsätze zu Grundfragen der Kunstwissenschaft, Berlin, 1998.

8. The Century Dictionary and Cyclopedia (CDC). New York: The Century Co, revised and enlarged, 1911 (orig. 1889). Vol. VIII, "screen".

9. Базен А. Что такое кино? Сб. статей. М.: Искусство, 1972.

10. Бергман И. Латерна магика. М.: Искусство, 1989.

11. Гинзбург С.С. Очерки теории кино. М.: Искусство, 1974.

12. Иоскевич Я.Б. Новые технологии и эволюция художественной культуры. Спб.: РИИИ, СПбГУКит, 2003.

13. Манович Л. Археология компьютерного экрана // Экранная культура. Теоретические проблемы: сб. статей / СПб.: «ДМИТРИЙ БУЛАНИН», 2012.

14. Мерш, Дитер. Мета/Диа. Два различных подхода к медиальному // Исследовательский центр медиафилософии, режим доступа: http://mediaphilosophy.ru/biblioteca/articles/mersh_dia/\#footnote-27

15. Основы музееведения. Учебное пособие. М.: Едиториал УРСС, 2005.

16. Флюссер В. За философию фотографии / Пер. с нем. Г. Хайдаровой. - СПб.: Изд-во С.-Петерб. ун-та, 2008.

17. Хухтамо Е. Элементы экранологии: к проблеме археологии медиа / Экранная культура. Теоретические проблемы: сб. статей / СПб.: «ДМИТРИЙ БУЛАНИН», 2012.

18. Юренев Р.Н. Чудесное окно: Краткая история мирового кино: Кн. для учащихся. - М.: Просвещение, 1983.

19. Е. В. Сальникова К вопросу о предыстории современной визуальной технической культуры // Культура и искусство. - 2012. - 2. - С. 53-61.

20. О.В. Красноярова Об истоках онтологизации медиа в феноменологии М. Мерло-Понти // Философия и культура. - 2012. - 1. - С. 23 - 29.

\section{References (transliterated):}

1. GereCh.Genealogyofthecomputerscreen//VisualCommunication.2006.No.5.DOI:10.1177/1470357206065306. Rezhim dostupa: http://vcj.sagepub.com/cgi/content/abstract/5/2/141

2. Huhtamo E. Elements of Screenology: Toward an Archaeology of the Screen // ICONICS: International Studies of the Modern Image. 2004. Vol. 7.

${ }^{22}$ Musser, Charles. Emergence of the Cinema: the American screen to 1907 // History of the American Cinema.

Berkeley; Los Angeles: University of California Press: 1994. Vol. 1. Chapter 1, "Towards a History of Screen Practice". 
3. Flusser V. Two approaches to the Phenomenon, Television / The New Television: A Public/Private Art / ed.by D.Davis and A. Simmons, Cambridge, Mass.: The Mitt Press, 1977.

4. Levie, Françoise. Étienne-Gaspard Robertson, la vie d'un fantasmagore, Longueuil et Bruxelles, Les Éditions du Préambule et Sofidoc, coll. «Contrechamp», 1990.

5. Musser, Charles. Emergence of the Cinema: the American screen to 1907 // History of the American Cinema. Berkeley; Los Angeles: University of California Press: 1994. Vol. 1. Chapter 1, "Towards a History of Screen Practice".

6. Oxford English Dictionary, II edition / ed.by J.A. Simpson and E.S.C. Weiner. Oxford: Clarendon Press, 1989.

7. Panofsky, Erwin. Die Perspektive als ,symbolische Form', in: ders., Aufsätze zu Grundfragen der Kunstwissenschaft, Berlin, 1998.

8. The Century Dictionary and Cyclopedia (CDC). New York: The Century Co, revised and enlarged, 1911 (orig. 1889). Vol. VIII, "screen".

9. Bazen A. Chto takoe kino? Sb. statei. M.: Iskusstvo, 1972.

10. Bergman I. Laterna magika. M.: Iskusstvo, 1989.

11. Ginzburg S.S. Ocherki teorii kino. M.: Iskusstvo, 1974.

12. Ioskevich Ya.B. Novye tekhnologii i evolyutsiya khudozhestvennoi kul'tury. Spb.: RIII, SPbGUKit, 2003.

13. Manovich L. Arkheologiya komp'yuternogo ekrana // Ekrannaya kul'tura. Teoreticheskie problemy: sb. statei / SPb.: «DMITRII BULANIN», 2012.

14. Mersh, Diter. Meta/Dia. Dva razlichnykh podkhoda k medial'nomu // Issledovatel'skii tsentr mediafilosofii, rezhim dostupa: http://mediaphilosophy.ru/biblioteca/articles/mersh dia/\#footnote-27

15. Osnovy muzeevedeniya. Uchebnoe posobie. M.: Editorial URSS, 2005.

16. Flyusser V. Za filosofiyu fotografii / Per. s nem. G. Khaidarovoi. - SPb.: Izd-vo S.-Peterb. un-ta, 2008.

17. Khukhtamo E. Elementy ekranologii: k probleme arkheologii media / Ekrannaya kul'tura. Teoreticheskie problemy: sb. statei / SPb.: «DMITRII BULANIN», 2012.

18. Yurenev R.N. Chudesnoe okno: Kratkaya istoriya mirovogo kino: Kn. dlya uchashchikhsya. - M.: Prosveshchenie, 1983.

19. E. V. Sal'nikova K voprosu o predystorii sovremennoi vizual'noi tekhnicheskoi kul'tury // Kul'tura i iskusstvo. $-2012 .-2 .-$ C. 53-61.

20. O.V. Krasnoyarova Ob istokakh ontologizatsii media v fenomenologii M. Merlo-Ponti // Filosofiya i kul'tura. 2012. - 1. - C. 23 - 29. 